\title{
AN UNUSUAL AIRWAY PROBLEM
}

\author{
G. R. ButliN, M.D., C.R.C.P.(C), G. R. SELLERY, M.D., F.R.C.P.(C), \\ AND W. E. SPOEREL, M.D., F.R.C.P.(C) *
}

ON VERY RARE OCCASIONS it may be necessary to ventilate each lung separately in association with major injuries to the trachea. A double lumen tube (e.g. Carlens tube) may be unsatisfactory if the defect in the trachea is of such a location and magnitude as to allow an air leak when the tracheal cuff is inflated. The following is a case report describing the management of the airway of a patient with a large tracheal defect about one centimeter above the carina, who required artificial ventilation.

Mr. W., a previously healthy man of 42 , sustained a severe chest injury on April 5, 1969. He required tracheostomy and artificial ventilation. On April 20, 1969, as his James tube was being removed, he sustained a massive haemorrhage from his tracheostomy stoma. ${ }^{1}$ This was partially controlled by digital pressure on the bleeding artery and the patient was taken to the operating room immediately, where the bleeding was controlled with great difficulty.

Severe bleeding recurred on four further occasions and each time was controlled by digital pressure and suturing of the bleeding vessel. However, associated with the difficulty of controlling the haemorrhage, the tracheal stoma progressively increased in size. By May 5 the patient had a large defect in the anterior wall of the trachea ending about one centimeter above the carina.

On May 6th, it was decided to attempt an aortic arch angioplasty under extracorporeal circulation. Controlled respiration, which would be required for a full sternal split thoracotomy, could not be achieved by using a cuffed tracheotomy or endotracheal tube, since the tracheal defect had extended too close to the carina. It was felt that the only way to solve this problem was to intubate each main bronchus separately with cuffed endobronchial tubes. After adequate topical anaesthesia of the larynx, a \#8 single lumen Gordon Greene tube was introduced with ease in the right main stem bronchus. Our intention was then to pass a long cuffed nasotracheal tube through the mouth and into the left main stem bronchus, but the patient's larynx would not allow even the smallest available cuffed tube $(6 \mathrm{~mm})$ to go through. Intubation of the left main stem bronchus directly through the tracheal stoma was ruled out as the surgeons felt that it would be incompatible with adequate surgical conditions. The bronchial cuff of the Gordon Greene tube was then inflated, the left lung allowed to collapse, and general anaesthesia with controlled ventilation of the right lung was conducted with clinically adequate oxygenation of the patient. Under cardiopulmonary bypass an aortic arch angioplasty and innominate artery ligation were then performed.

At the end of the procedure an $8 \mathrm{~mm}$ endotracheal tube was introduced

-Department of Anaesthesia, University of Western Ontario, London, Ontario. 


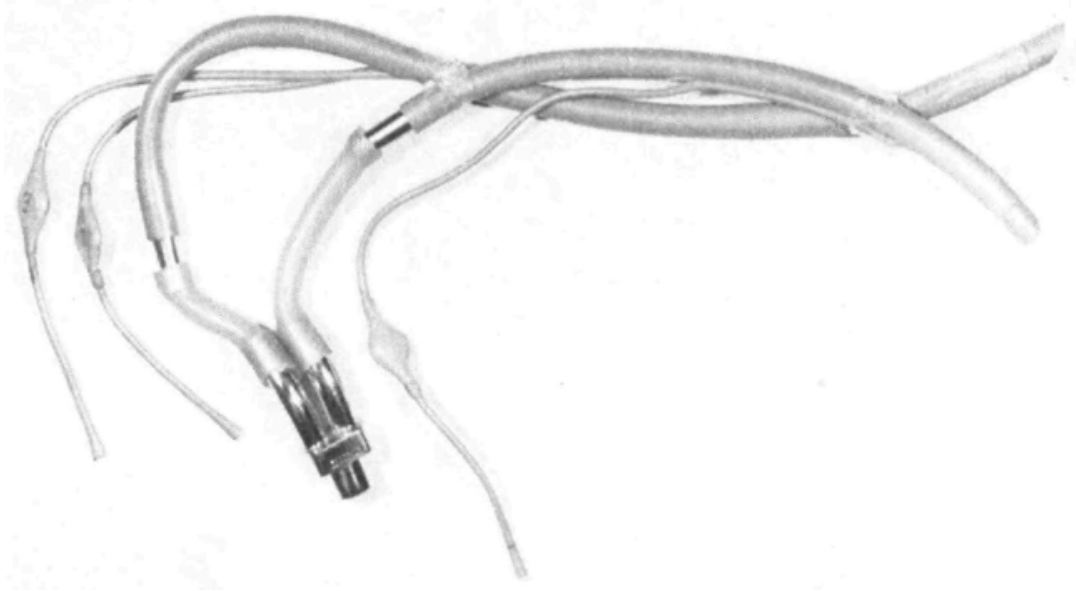

Figure 1. Photograph of endobronchial tubes in approximate position as they were when used in patient. The Carlens connector is attached.

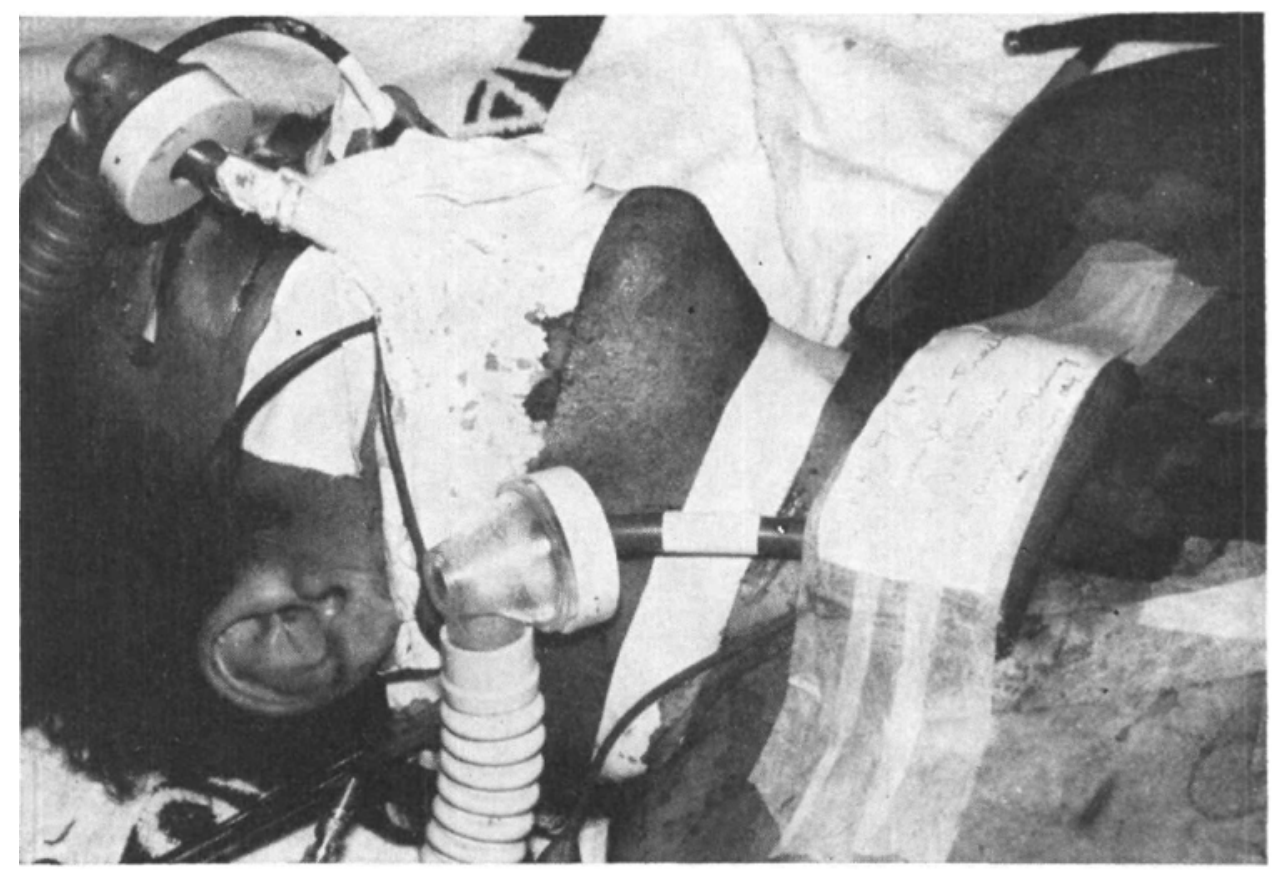

Frgure 2. Patient breathing spontaneously. The left endobronchial tube emerges through the tracheotomy site; the right endobronchial tube through the mouth. 


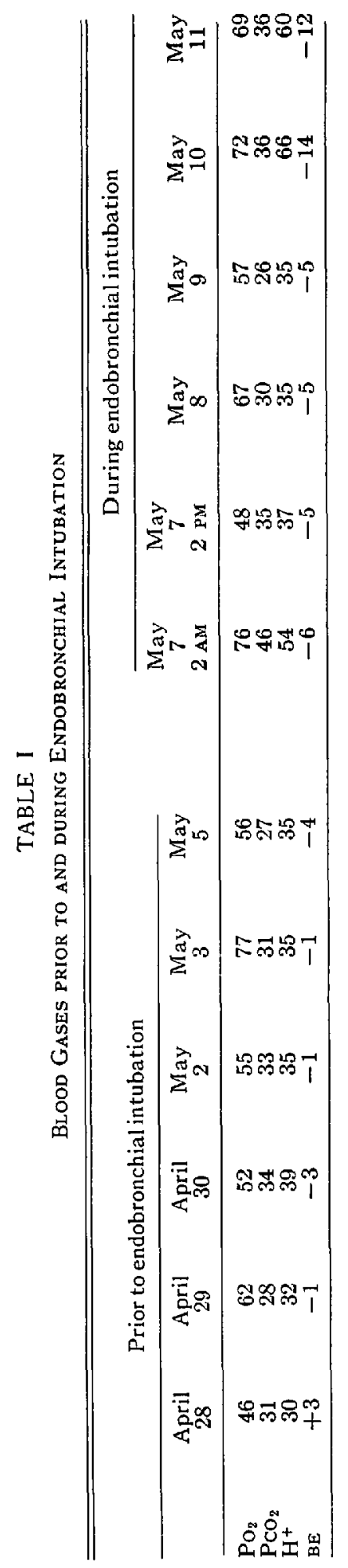




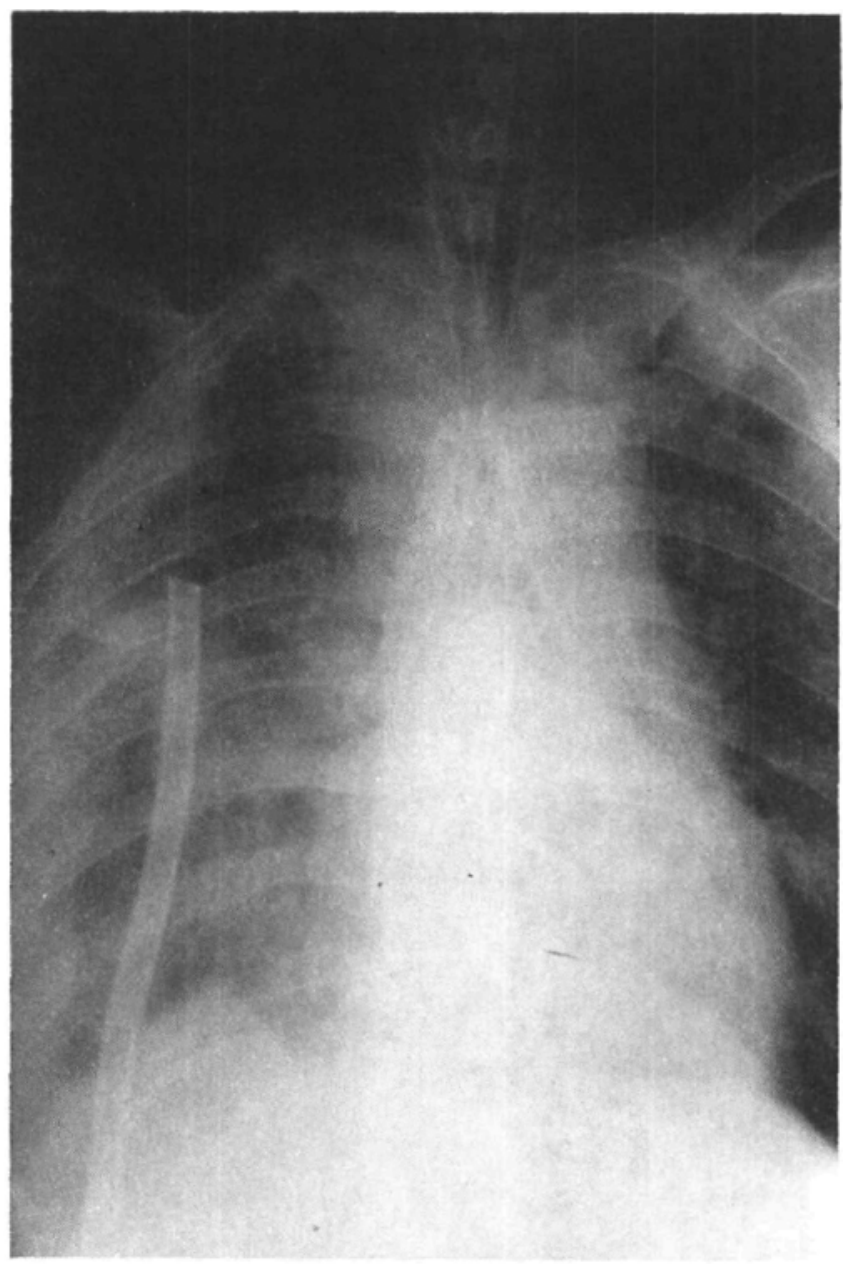

Frgure 3. Supine PA chest $\mathrm{X}$-ray shows adequate positioning of the endobronchial tubes.

through the tracheotomy stoma into the left main stem bronchus. In order to avoid further trauma to the trachea, the orotracheal Gordon Greene tube was left in place. The left endobronchial tube, introduced through the tracheostomy, and the right endobronchial, passed through the larynx, were then attached to a Carlens connector and the patient was ventilated overnight with a Mark 10 Bird respirator. (Fig. 1). This tube arrangement was left in place for five days with the patient on and off the respirator (Fig. 2). Suctioning through the endobronchial tubes presented no problems.

Follow-up analyses of blood gases showed borderline ventilation, but these were essentially not different from those obtained prior to the use of endobronchial intubation (Table I). The positioning of the tubes was checked daily by chest X-rays (Fig. 3 ).

The patient died on May 11 due to overwhelming infection (mediastinitis). 
The tubes were left in place until an autopsy was performed. Post mortem examination showed the side opening of the Gordon Greene tube just opposite to the right upper lobe bronchus. The tip of the left endobronchial tube was situated $1.5 \mathrm{~cm}$ above the opening of the left upper lobe bronchus. The bronchial mucosa was reddened, but there were no ulcerations.

\section{SUMMARY}

In a patient with a large defect of the anterior tracheal wall, a technique of a bilateral endobronchial intubation was used. With a Gordon Greene tube in the right main bronchus and a Magill tube in the left main bronchus, maintenance of an airway and ventilation of both lungs was possible for five days.

\section{RÉSUMÉ}

Chez un malade souffrant d'une défectuosité grave de la paroi antérieure de la trachée, on a utilisé une technique d'intubation endobronchique bilatérale. A l'aide d'un tube de Gordon Greene dans la bronche souche droite et d'un tube Magill dans la bronche souche gauche, on a pu maintenir un libre passage de l'air et ventiler les deux poumons durant cinq jours.

\section{REFERENCE}

1. Ivankovic, A. D.; Thomsen, S.; \& Rattenborg, C. C. Fatal Haemorrhage from the Innominate Artery after Tracheostomy. British Journal of Anaesthesia. 41: 450 (1969). 